daily recording of ECG, blood pressure, weight, and\% body-water (bioimpedance), Stroop test (hepatic encephalopathy (HE) assessment), and self-reported well-being and food/fluid/alcohol intake. Data was Blue-toothed via a secure server to the CirrhoCare ${ }^{\circledR}$-App, which had 2-way patient-physician communication. Hepatologists evaluated daily data and facilitated interventions as required. A matched control cohort $(n=20)$ with advanced cirrhosis was observed in parallel.

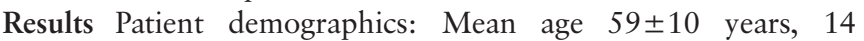
male, main etiology alcohol (75\%); 75\% Child-Pugh class B. Fifteen patients $(75 \%)$ showed good compliance, $(\geq 4$ readings/ week), 2 had moderate compliance (2-4/week), and 3 had poor compliance $(<2 /$ week). In a usability questionnaire scored $1-10$, the median score was $\geq 9$ for any given question.

Mean follow-up was $10.1 \pm 2.4$ weeks. Amongst CirrhoCare ${ }^{\circledR}$ managed patients, 1 died and 1 received a liver transplant. Eight readmissions occurred in 5 different patients: 3 due to $\mathrm{HE}, 1$ to acute-kidney injury (AKI), 1 to both AKI and HE, and 3 in the same patient to rectal bleeding. The median readmission lasted 5 (IQR 3.5-11) days, and none was $>14$ days. Except for the acute bleeds, we identified early signs of decompensation in all cases, e.g. failed Stroop test, hypotension or reduction/gain in body fluid (weight), and facilitated 2 short hospitalizations of the 8 total readmissions.

Based on early signs of decompensation, we contacted patients on 16 other occasions, guiding intervention and likely preventing further readmissions as confirmed by an independent physician panel. Two controls died during follow-up, and there were 13 readmissions in 8 patients, lasting median 7 (IQR 3-15) days with four admissions $>14$ days. They had 6 unplanned paracenteses compared to 1 in CirrhoCare ${ }^{\circledR}$-managed patients.

Conclusions CirrhoCare ${ }^{\circledR}$ 's novel, multimodal, home-monitoring in patients with advanced cirrhosis is feasible with excellent patient engagement, and prompts early diagnosis of decompensating events and their intervention; and hospital admissions are fewer and shorter in duration than in controls. We propose the application of CirrhoCare ${ }^{\circledR}$ for assisted, specialist, community management of advanced cirrhosis.

\section{P099 SERUM GAMMA GLUTAMYL TRANSFERASE ( $\gamma$ GT) - A PROGNOSTIC BIOMARKER FOR DETRIMENTAL NEUTROPHILS IN PATIENTS WITH HEPATOCELLULAR CARCINOMA (HCC)?}

${ }^{1}$ Marcello Kadharusman, 'Misti McCain, 'Joao Mauricio, 'Maja Laszczewska, 'Jack Leslie, ${ }^{1}$ Daniel Geh, ${ }^{1}$ Derek Mann, ${ }^{1}$ Ruchi Shukla, ${ }^{1,2}$ Helen L Reeves*. ' Newcastle University, Newcastle upon Tyne, UK; ${ }^{2}$ Newcastle upon Tyne Hospitals NHS Foundation Trust, Newcastle upon Tyne, UK

\subsection{6/gutjnl-2021-BASL.107}

Background Deaths from HCC are rising dramatically in the UK. Immuno-oncology therapies herald a new era of treatment, but only a minority of patients respond. Elevated circulating neutrophils are associated with a poor prognosis, although mechanisms are poorly understood and clinically useful biomarkers of 'poor prognostic neutrophils' are lacking. Serum $\gamma$-GT recognised as a biomarker of a neutrophil inflamed liver microenvironment. As a membrane-bound enzyme it promotes intracellular glutathione, limiting cellular damage. It may also promote cancer cell survival.

Methods Serum $\gamma$-GT associations were explored in 300 patients with HCC. Correlations with tissues expression and CD66b +ve neutrophils were explored in a subset of 50 patients.

Results There were highly significant positive correlations $(<0.01)$ between serum $\gamma$-GT and deteriorating ECOG PST and presence of portal vein thrombosis (PVT). Serum $\gamma$-GT correlated negatively with albumin (Pearson -0.134, $\mathrm{p}=0.020$ ) and positively with bilirubin $(0.124, \mathrm{p}=0.032)$, neutrophils (0.221, $\mathrm{p}<0.0001)$ and platelets $(0.171, \mathrm{p}=0.003)$. In a multivariate cox regression analysis, ECOG PST, albumin, PVT, platelets and $\gamma$-GT were independently associated with poorer survival. $\gamma$-GT expression is typically on the canalicular surface of hepatocytes. In a subset of patients with tissues available, immunohistochemistry confirmed an elevation in expression intensity in up to $50 \%$ of patients with HCC compared to cirrhotic controls without cancer. In addition, aberrant membranous and cytoplasmic $\gamma$-GT expression was acquired in $30 \%$ of cancer cases - in tumour as well as peri-tumour liver. It was the cytoplasmic $\gamma$-GT in peri-tumour liver that correlated significantly with declining PST, Childs Pugh score and PVT. Tissue neutrophils were detected with CD66b immunohistocehmistry. These were elevated in peri-tumour areas, associated with poorer response to treatment and outcome. Elevation in $\mathrm{CD} 66 \mathrm{~b}+$ neutrophils was associated with the aberrant cytoplasmic $\gamma$-GT (See figure 1). Studies are ongoing, but $\mathrm{CD} 66 \mathrm{~b}$ positive neutrophils in the peri-tumour area correlated with serum $\gamma$-GT in a highly significant fashion $(0.838$, $\mathrm{p}=0.001$, Spearmans Rho, $\mathrm{n}=11$ ).

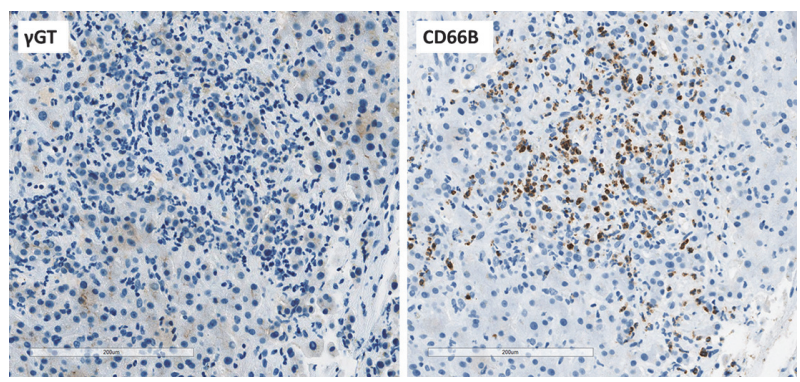

Abstract P099 Figure 1 Aberant cytoplasmic $\gamma \mathrm{GT}$ in peri-tumour liver, with CD66b+ neutrophils

Conclusion/Discussion Serum $\gamma$-GT correlates significantly with invasive/advanced HCC, deteriorating ECOG PST and circulating neutrophils and is independently associated with poorer survival. In tissues, aberrant cytoplasmic $\gamma$-GT is present in the peri-tumour liver, enriched with CD66b positive neutrophils, both associated with progressive tumour features and serum $\gamma$-GT. Ongoing studies will define the neutrophil phenotype associated with aberrant $\gamma$-GT expression, while exploring serum $\gamma$-GT as a predictive biomarker, guiding immuno-oncology combination therapies. Combinations targeting pro-tumour neutrophils in combination with those targeting $\mathrm{T}$ cells may increase responses to immunooncology therapies. 